\title{
Effect of Fluorescent Particle Size on the Modulation Efficiency of Ultrasound-Modulated Fluorescence
}

\author{
Yuan Liu, ${ }^{1,2}$ Baohong Yuan, ${ }^{1,2}$ and Joseph Vignola ${ }^{3}$ \\ ${ }^{1}$ Department of Bioengineering, The University of Texas at Arlington, Arlington, TX 76019, USA \\ ${ }^{2}$ Joint Biomedical Engineering Program, The University of Texas at Arlington, and The University of Texas Southwestern \\ Medical Center at Dallas, TX 75390, USA \\ ${ }^{3}$ Department of Mechanical Engineering, The Catholic University of America, Washington, DC 20064, USA
}

Correspondence should be addressed to Baohong Yuan, baohong@uta.edu

Received 15 July 2011; Revised 15 September 2011; Accepted 15 September 2011

Academic Editor: Nanguang Chen

Copyright ( $) 2012$ Yuan Liu et al. This is an open access article distributed under the Creative Commons Attribution License, which permits unrestricted use, distribution, and reproduction in any medium, provided the original work is properly cited.

To investigate whether the size of fluorescent particles affects the modulation efficiency of ultrasound-modulated fluorescence (UMF), we measured UMF and DC (direct current) signals of the fluorescence emission from four different-sized fluorescent particles: (1) three carboxylate-modified fluorescent microspheres (FM) with diameters of $20 \mathrm{~nm}, 200 \mathrm{~nm}$, and $1.0 \mu \mathrm{m}$ and (2) streptavidin-conjugated Alexa Fluor 647 with a diameter of approximately $5 \mathrm{~nm}$. The UMF and DC signals were simultaneously measured using a broadband lock-in amplifier and a narrowband amplifier, respectively. The ratio of the UMF strength to the DC signal strength is defined as the modulation efficiency. This modulation efficiency was then used to evaluate the effects of fluorophore size and concentration. Results show that the modulation efficiency was improved by approximately a factor of two when the size of the fluorescent particles is increased from $5 \mathrm{~nm}$ to $1 \mu \mathrm{m}$. In addition, the linear relationship between the UMF strength and ultrasound pressure (observed in our previous study) was maintained regardless of the fluorescent particle sizes.

\section{Introduction}

Tissue fluorescence imaging has been well developed and widely used because of its high sensitivity and specificity $[1,2]$. Fluorescence techniques can provide unique tissue physiological information when compared with other noninvasive imaging modalities (ultrasound, magnetic resonance imaging, computed tomography, etc.) and are sensitive to tissue microenvironments, such as tissue $\mathrm{pH}$, temperature, and gas/ion concentrations. Also, they are relatively cost efficient, flexible in imaging probes selection (from organic dyes, to quantum dots, and to nanoparticles or microparticles), highly sensitive to imaging probes ( $\mathrm{fM}-\mathrm{nM}, 10^{-15}$ $10^{-9}$ mole/liter), and nonionizing radiative [1-3]. Commonly used high-resolution fluorescence microscopy faces a fundamental challenge due to tissue's strong optical scattering, which typically limits penetration depth to a few hundred micrometers [3]. Techniques used to image deep tissue at ranges of millimeters or centimeters, such as fluorescence diffuse optical tomography (FDOT) [4], take advantage of diffused photons that have been scattered many times before being detected. These diffused photons can penetrate biological tissue up to tens of millimeters at the red or near infrared (NIR) region [3] at the expense of spatial resolution (limited to $\sim 1-5 \mathrm{~mm}$ ) [4].

Ultrasound-modulated fluorescence (UMF) has been proposed to increase spatial resolution while maintaining imaging depth [5]. This is possible because ultrasonic scattering is two to three orders of magnitude less than optical scattering in biological tissues [3]. The concept of UMF is similar to ultrasound-modulated optical tomography (UOT) [6-8], although their mechanisms may be different $[9,10]$. In UMF, a focused ultrasound beam is used to tag the diffused fluorescence photons in the focal zone. By detecting and analyzing the tagged (or modulated) fluorescence photons, one can quantify the fluorescence properties in the ultrasound focal zone with ultrasonic spatial resolution that is usually much higher than the resolution of FDOT [11-15]. Recently, several research groups have investigated UMF both theoretically $[5,10]$ and experimentally [11-15]. The modulation efficiency of UMF is limited and has to be significantly improved for applications in biomedical imaging [11-15]. 
Kobayashi et al. reported a modulation efficiency in UMF adequate for tomographic imaging in a scattering medium using relatively large fluorescent microspheres $(\sim$ microns in diameters) [11]. However, their results seem to disagree with our recently reported experimental data collected using relatively small organic fluorescent dyes (nanometers in diameters) $[12,13]$ and that of Hall et al. $[14,15]$ (see the detailed discussion about the disagreements in [12]). Therefore, the question of whether relatively large fluorescent particles $(\sim$ microns $)$ can reliably and significantly improve the modulation efficiency of UMF is of interest.

The study of the effect of particle size on the modulation efficiency can also lead to a better understanding of the underlying UMF mechanisms. The modulation mechanisms of UMF are quite different from those of UOT [10] due to the intrinsic incoherent properties of fluorescence emission in tissue. While UOT has been well studied using coherent light [9], the modulation mechanisms of UMF are still poorly understood due to inconsistent data in literature [11-15]. Previously, we have proposed several possible mechanisms for UMF [10]. The most plausible one is that the UMF signal is caused by periodic oscillations of the fluorophore concentration that are generated by ultrasonic pressure wave [10]. We have reported a supporting evidence of this mechanism based on a recent experimental data $[12,13]$. Although this mechanism can only provide a relatively small modulation efficiency (typically $10^{-4}-10^{-6}$ ) in a scattering medium, it exists in all types of fluorophores from small nanoscale fluorescent molecules to relatively large nano-/microscale fluorescent particles $[10,12,13]$. Intuitively, one might think that it is easier to modulate large fluorescent microparticles with an ultrasound wave because larger particles may be ultrasonically oscillated more efficiently; besides, each microparticle can encapsulate more fluorescent molecules. Potentially, this could provide a means to improve the modulation efficiency of UMF. If this hypothesis is proved true, the next question would be how much improvement can be achieved and is it sufficient for biomedical imaging applications? To address these basic questions, UMF modulation efficiencies for four communally available fluorescing particles were measured and discussed in this study.

\section{Methods}

Our previous studies have shown that the UMF signal is difficult to quantify in a scattering medium due to its poor signalto-noise ratio [13]. As a result, water was used as the medium in this study. Three fluorescent microspheres with diameters of $20 \mathrm{~nm}, 200 \mathrm{~nm}$, and $1.0 \mu \mathrm{m}$ were tested in this study. To further reduce the particle size, Alex Fluor 647 conjugated with streptavidin $(\sim 1 \mathrm{~nm}$ in diameter for the dye molecule and $\sim 5 \mathrm{~nm}$ in diameter for the streptavidin $[16,17])$ was selected as a sample in comparison. The UMF signal was measured using a broadband lock-in amplifier, and simultaneously the fluorescent DC signal was measured using a narrowband amplifier. With both measurements calibrated, the ratio of these two quantities is recognized as the modulation efficiency. A consistent experimental setup was used for all the samples in the study so that results for different samples could be compared. The modulation efficiency is evaluated as a function of the fluorescent particle size.

The experimental setup is shown in Figure 1. The excitation light was produced by a fiber-coupled diode laser (57ICS064/SP/HS, Melles Griot) with a wavelength of $638 \mathrm{~nm}$. The monochromatic light is guided via a polarization maintaining fiber (57FTP602/FC/1.0 Melles Griot, core diameter: $4 \mu \mathrm{m}$ ) to a collimating lens (F240FC-B, Thorlabs). The power density of the laser was controlled as $411 \mathrm{~mW} / \mathrm{cm}^{2}$. A silicon tube (FT, Instech Laboratories, inner diameter: $0.79 \mathrm{~mm}$, outer diameter: $2.39 \mathrm{~mm}$ ) was positioned in a water-filled container $(40(x) \times 120(y) \times$ $120(z) \mathrm{mm}$ ) and orientated parallel to the $y$-axis. An ultrasound transducer (UST, V314-SU-F-1.00-IN-PTF, Olympus NDT, center frequency: $1 \mathrm{MHz}$ ) was focused on the intersection of the laser beam and the FT (see Figure 1). The UST was driven by a continuous sinusoidal signal with a frequency of 1.0 MHz that was generated from a function generator (FG, Agilent 33120A, Agilent Tech, Calif, USA) and amplified by a power amplifier (PA, $7600 \mathrm{M}$, Krohn-Hite, Mass, USA). The peak pressure at the transducer focus was measured by a hydrophone (HNP-0200, Onda Corporation, Calif, USA) and preamplifier (AH2010, Onda Corporation, Calif, USA). Two lenses (L1 and L2, AC254-030-A1, Thorlabs) were used to collect the emitted fluorescence. Long-pass emission filters (EmF, BLP01-635R, Semrock, cutoff wavelength: $650 \mathrm{~nm}$ ) were employed to reject the excitation light. The emission photons were focused on two adjacent channels of a 16-channel photomultiplier tube (PMT, R5900U-01L16, Hamamatsu, Japan) at the central area of this PMT to monitor the UMF and the fluorescence DC signals. The photocurrent from one channel was converted to a voltage signal using a transimpedance amplifier (TIA, 313A-1-1 pF, Analog Modules, Longwood, Florida), and its output was directed to a broadband lock-in amplifier (LIA, SR844, Stanford Research Systems, Calif, USA). The synchronized TTL (transistor-transistor logic) signal of the function generator was used as the reference signal for the LIA. The output of the LIA represents the UMF strength. The photocurrent from the second channel was directly connected to an oscilloscope (OS, TDS 3032B, Tektronix, Oregon). The input impedance of the oscilloscope was selected to be 1 Mega Ohms so that an appropriate gain can be obtained with a limited bandwidth. This signal was used to record the DC level of the fluorescence. Four sets of measurements were conducted for each sample using a new solution and tube. In each set of measurements, the UST was scanned for 3 times along the $x$-axis using a linear translation stage (PT1, Thorlabs, NJ, USA), and the DC and UMF signals were recorded at each selected position of the UST. The average and the standard derivation were calculated and displayed based on all the measurements.

Before any measurements were conducted, the tube was ultrasonically located by connecting the UST to a pulse generator/receiver (5077PR, Olympus NDT, Waltham, Mass, USA). The peak-to-peak voltage of the ultrasonic echoes were recorded at selected positions when scanning the UST along the $x$-axis. The background voltage, which was mainly caused by the electronic interference from the UST driven 


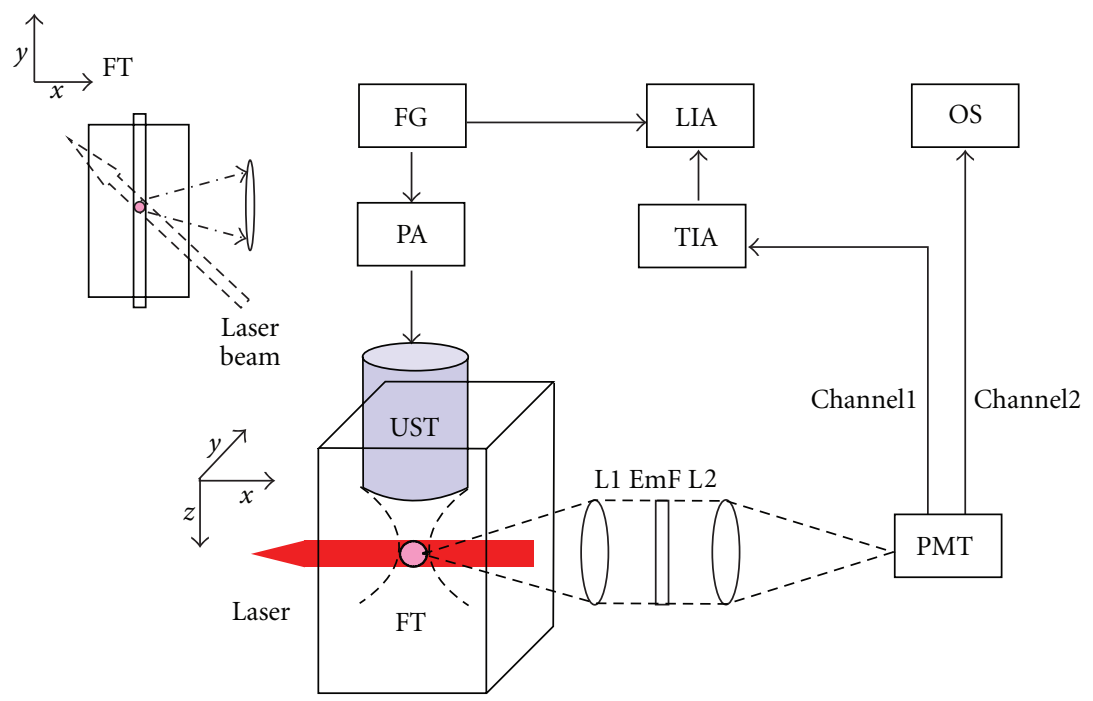

FIGURE 1: Schematic of the measurement system. FG: function generator; PA: power amplifier; UST: ultrasound transducer; FT: fluorescent tube; L1-L2: two lenses; EmF: emission filters; PMT: photomultiplier tube; TIA: transimpedance amplifier; LIA: lock-in amplifier; OS: oscilloscope. The inset represents the top view of the container and FT.

signal, was recorded when the excitation laser beam was blocked. This background voltage is independent of the UST position and can be subtracted from the measured UMF signals.

The UMF signal and the fluorescent DC signal were measured as a function of the fluorophore concentration. The concentrations of the Alexa Fluor 647 solutions (S21374, Invitrogen) were $0.025,0.05,0.1$, and $0.2 \mathrm{mg} / \mathrm{mL}$, and the concentrations for the fluorescent microsphere solutions (FM, F8782, F8806, F8816, FluoSpheres, Invitrogen) were $0.5,1.0,2.0$, and $4.0 \mathrm{mg} / \mathrm{mL}$. New clear tubes were used for each fluorescent sample to avoid the contamination from the previous sample. The effect of the tube autofluorescence is undetectable at the $638 \mathrm{~nm}$ excitation wavelength and can be ignored. This has been verified by detecting the UMF signal when filling the tube with water. Our results showed that the detected signal is almost a constant and independent of the location of the ultrasound transducer, which implies that no UMF signal can be detected when no fluorescent solution is present. Ideally, the linear fits of the experimental data should pass through the origin, implying that both the UMF and the fluorescence DC signals should be zero when the concentration is zero. In practice, this is not always true because of the existence of background interference and/or instrument bias. Therefore, the intercepts of the linearly fitted data were subtracted during the data processing. The ratio of the intercept-subtracted UMF signal to the intercept-subtracted DC signal is defined as the modulation efficiency. The absolute value of the modulation efficiency (the ratio) may not represent the true physical meaning of the modulation depth because the gains of the two channels may not be the same. However, because the measurement system and all experimental parameters were maintained the same for all the samples, the relative values of the defined modulation efficiency for all the samples can be compared.

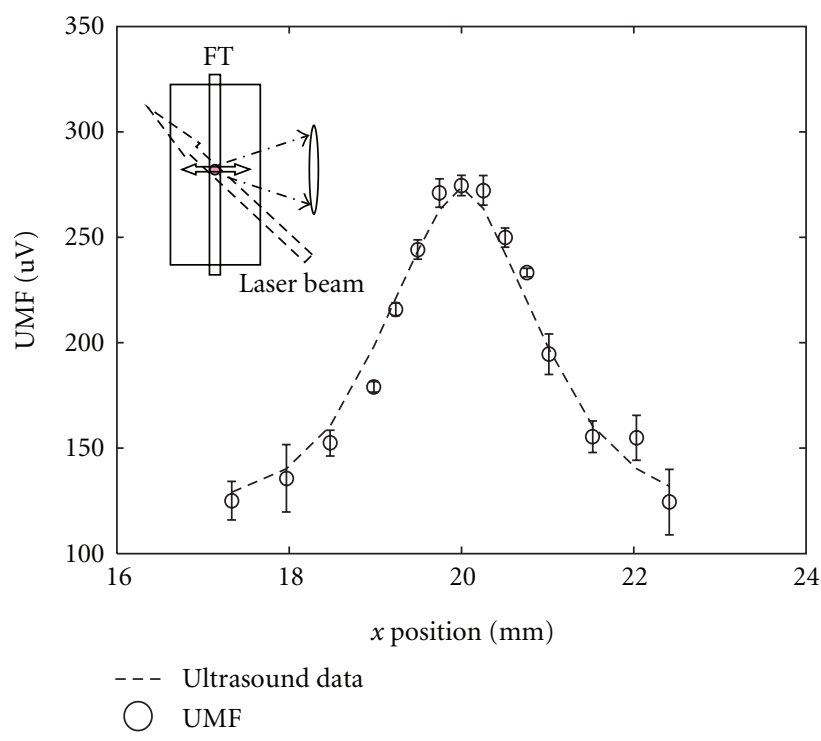

FIgURE 2: The circles with error bars represent the measured UMF signals from one of the fluorescent microsphere solutions (with the diameter of $200 \mathrm{~nm}$ and the concentration of $1.0 \mathrm{mg} / \mathrm{mL}$ ) as a function of the $x$ position of the UST. The dashed line shows the rescaled ultrasound signal. The inset represents the measurement configuration, and the doubled arrow shows the UST scan line. $P_{\text {fspp }}=180 \mathrm{KPa}$.

\section{Results and Discussion}

Figure 2 displays the measured UMF signals (the circles with error bars) from the tube filled with $200 \mathrm{~nm}$ diameter FM solution $(1.0 \mathrm{mg} / \mathrm{mL})$ after scanning the UST along the $x$ axis. The inset shows the measurement configuration. The ultrasound data was linearly rescaled as to display both the ultrasound data and the UMF data in the same figure. The origin of the $x$-axis is defined as the left edge of the 


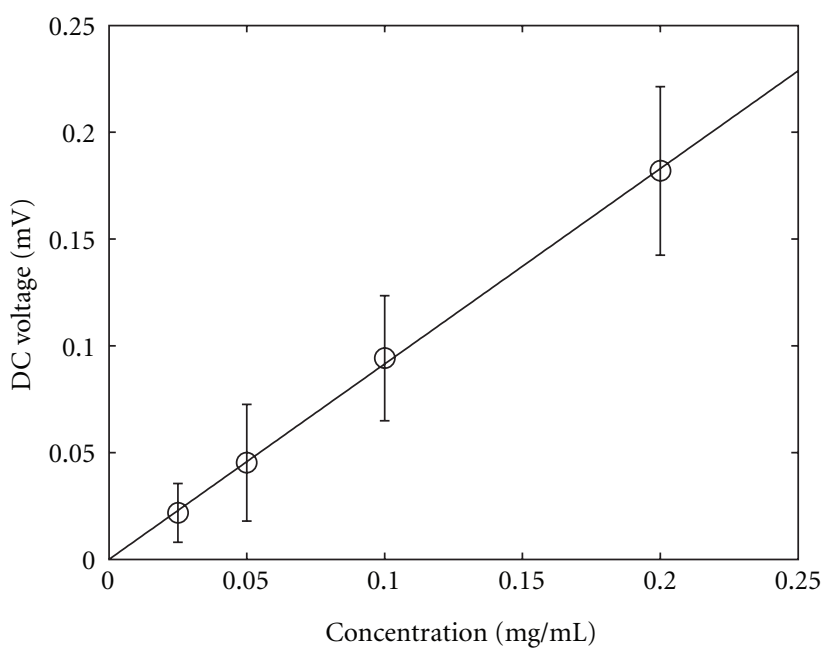

Alexa fluor 647

__ Linearly fitted data

(a)

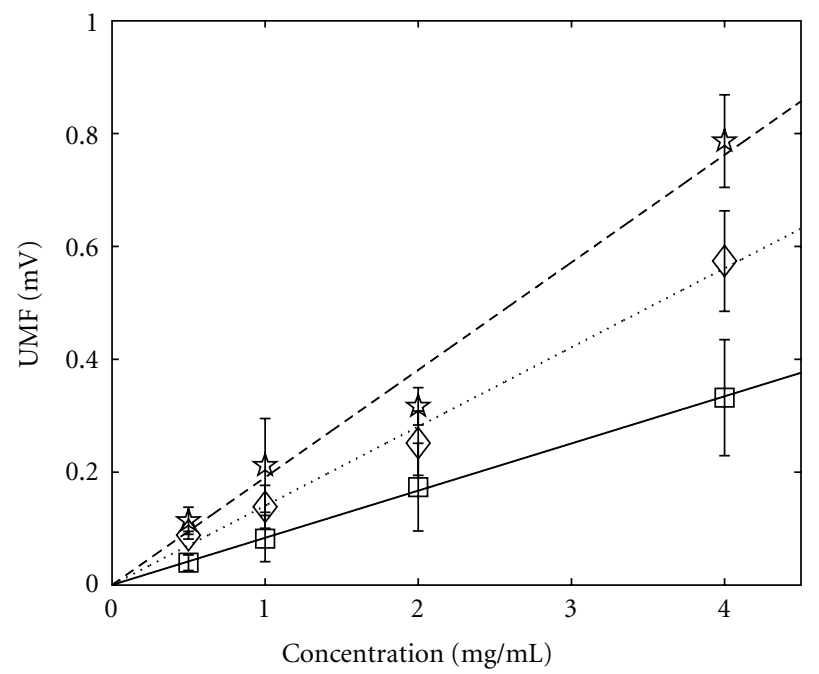

$\square$ FM $20 \mathrm{~nm}$

$\diamond \mathrm{FM} 200 \mathrm{~nm}$

家 $\mathrm{FM} 1 \mu \mathrm{m}$

(c)

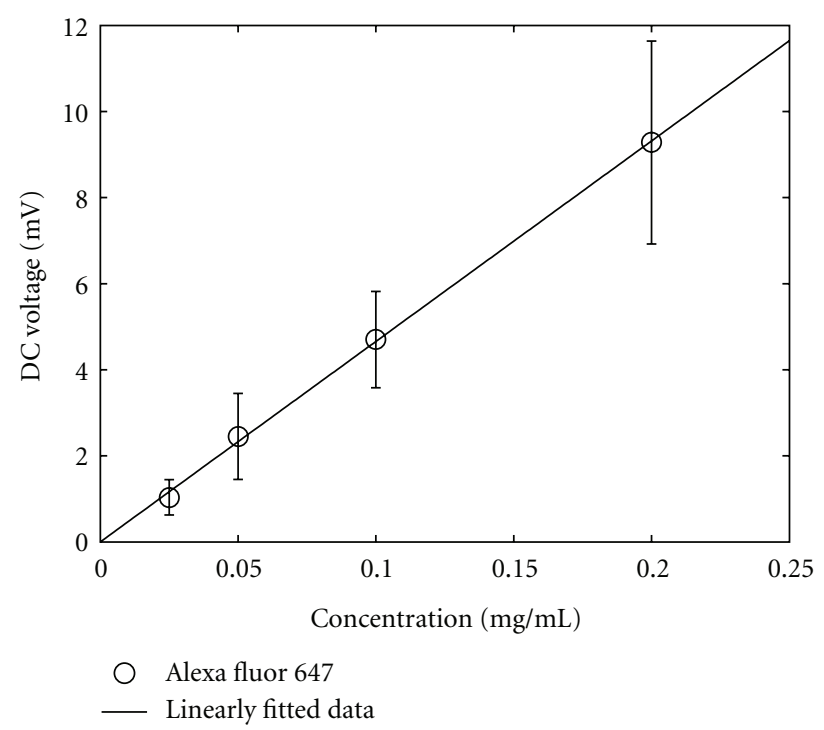

(b)

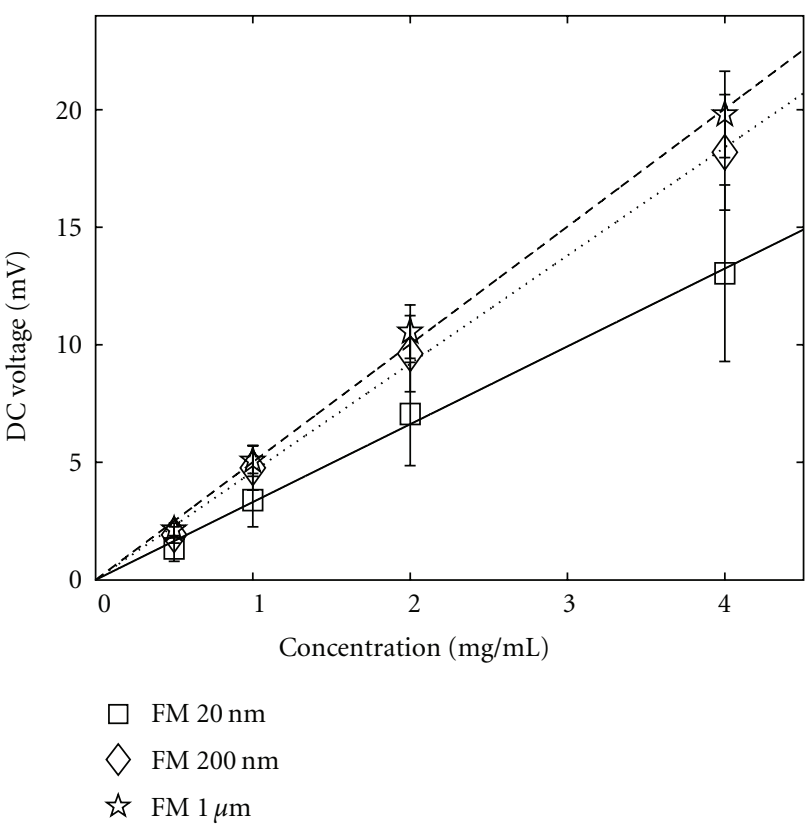

(d)

FIgURE 3: UMF and DC signals as a function of fluorophore concentration when $x=20 \mathrm{~mm}, y=0 \mathrm{~mm}$, and $P_{\text {fspp }}=180 \mathrm{KPa}$. (a) and (b) show the UMF signal and DC signal of streptavidin-conjugated Alexa Fluor 647, respectively. The circles with error bars represent the experimentally measured data, and the solid line is the linearly fitted data. (c) and (d) show the UMF signal and DC signals from the fluorescent microsphere solutions, respectively. The squares, diamonds, and stars represent the experimentally measured data. The solid, dotted, and dashed lines are the linearly fitted data.

tank, and the tube is located at the center of the tank $(x=20$ and $y=0 \mathrm{~mm})$. The peak pressure applied at the transducer focus $\left(P_{\mathrm{fspp}}\right)$ is $180 \mathrm{KPa}$. Figure 2 indicates a correlation between the UMF signal and the ultrasound. The modulation efficiency of different fluorophores is based on the peak values of the UMF signals.

Figure 3 shows the peak values of the measured UMF and DC signals from the four fluorescent particle solutions as a function of fluorophore concentration when
$P_{\text {fspp }}=180 \mathrm{KPa}$. Figures 3(a) and 3(b) show the UMF signal and the DC signal from the streptavidin-conjugated Alexa Fluor 647, respectively. The circles with error bars represent the experimentally measured data, and the solid lines are the linearly fitted data. Similarly, Figures 3(c) and 3(d) show the UMF signal and the DC signals from the three fluorescent microsphere solutions, respectively. The squares, diamonds, and stars represent the experimentally measured data. The solid, dotted, and dashed lines represent linear fits 


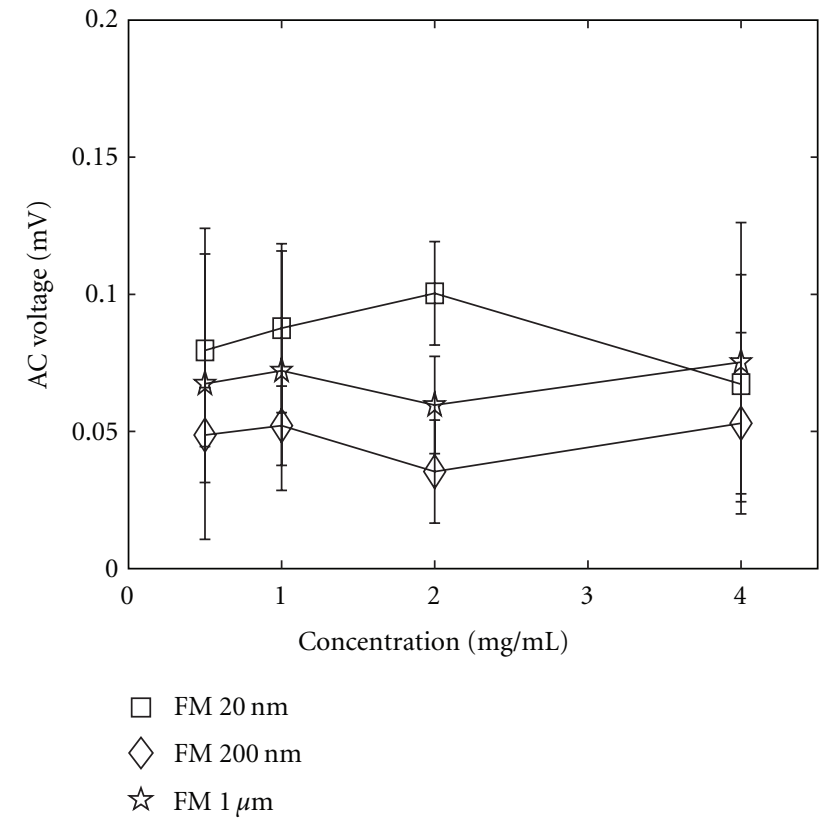

Figure 4: Ultrasound-modulated signal (AC voltage) of the scattered excitation light from the microsphere solutions as a function of concentration, when $x=20 \mathrm{~mm}, y=0 \mathrm{~mm}$, and $P_{\text {fspp }}=180 \mathrm{KPa}$. The squares, diamonds, and stars represent the experimentally measured data.

of the data. Figures 3(b) and 3(d) show a linear relationship between the DC signal and fluorophore concentrations from all the four samples. These linear relationships imply that the concentrations chosen in this study are appropriate and the inner filter effect or self-quenching is negligible.

To investigate the possibility of ultrasound-induced modulation of excitation light, we also detected the AC signal of the excitation light by blocking the emission light. Figure 4 shows the ultrasound-modulated signal (AC voltage) of the excitation light as a function of microsphere concentration. The squares, diamonds, and stars represent the experimentally measured data. For all three microsphere solutions, the AC signals do not vary significantly with the concentrations in the adopted range. Also, the differences of signal strengths from different particle solutions are not significant. Interestingly, the signal from 1.0 micron particles is higher than that from $200 \mathrm{~nm}$ particles. However, both are lower than that of $20 \mathrm{~nm}$ particles solution. These results indicate that modulation of the excitation light is complicated and may originate from various mechanisms [9]. Although the dominant modulation mechanism is difficult to be judged from the data shown in Figure 4, a few conclusions can be drawn based on our current and previous studies $[5,10,12$, 13]. First, the modulated fluorescence signal can be clearly observed in a solution without scatterers, such as rhodamine $B$ aqueous solution where the fluorophores are smaller than $1 \mathrm{~nm}$ in diameters and do not contribute to light scattering [13]. It implies that the scatters are not necessary to generate UMF signal. Secondly, the modulation of the excitation light is not well correlated with fluorophore concentration and particle size. In contrast, the observed UMF does. This result

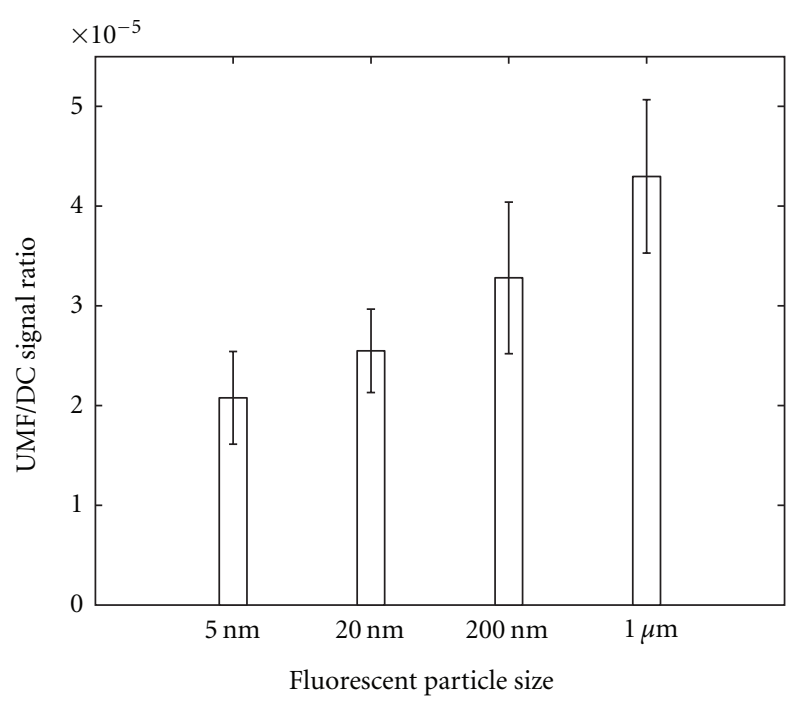

Figure 5: Modulation efficiencies of the four types of samples with diameters of $5 \mathrm{~nm}$ (as an approximation to streptavidin-conjugated Alexa Fluor 647), $20 \mathrm{~nm}, 200 \mathrm{~nm}$, and $1.0 \mu \mathrm{m}$.

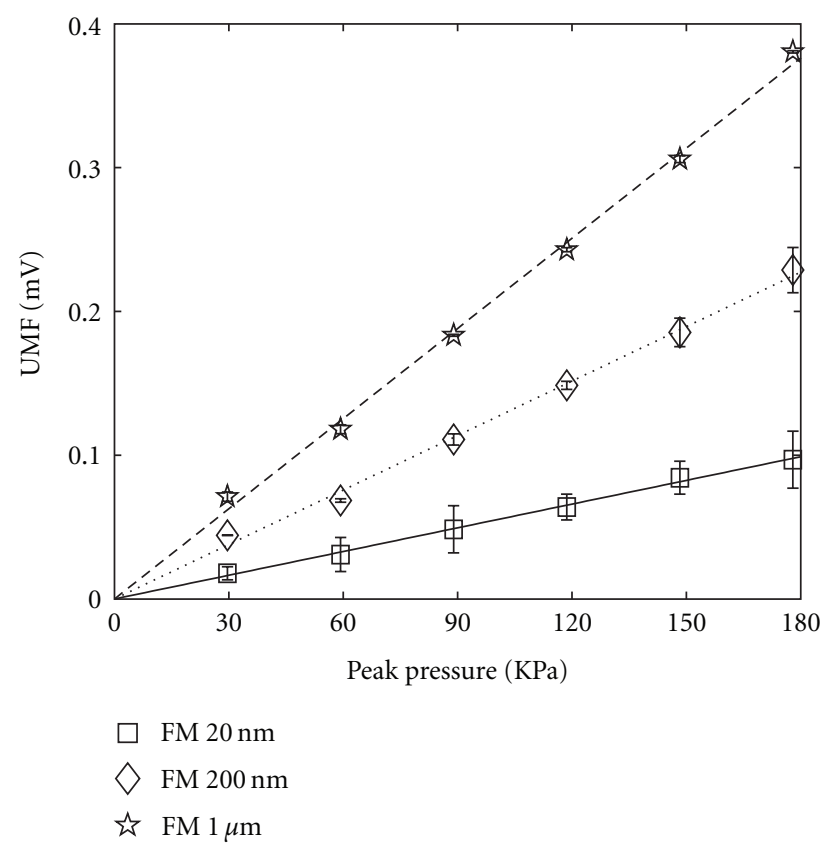

FIGURE 6: UMF signals from the fluorescent microsphere solutions with the concentration of $1.0 \mathrm{mg} / \mathrm{mL}$ as a function of $P_{\mathrm{fspp}}$ (after the subtraction of the electronic interference) when $x=20 \mathrm{~mm} y=$ $0 \mathrm{~mm}$. The squares, diamonds, and stars with error bars represent the experimentally measured data from the three different-sized microsphere solutions $(20 \mathrm{~nm}, 200 \mathrm{~nm}$, and $1.0 \mu \mathrm{m})$, respectively. The solid, dotted, and dashed lines are the linear fits.

may indicate that the modulation of the fluorophore concentration is dominant in which the unmodulated excitation light excites the modulated fluorophores.

Figure 5 shows the calculated modulation efficiencies (the ratio of the UMF signal to the fluorescence DC signal) of three FM and the streptavidin-conjugated Alexa Fluor 647 
fluorescent probes. The modulation efficiency increases with increasing particle size for fluorescent microsphere solutions. The modulation efficiency of the fluorescent microsphere sample with a diameter of $1.0 \mu \mathrm{m}$ is $\sim 1.3$ times higher than that of the $200 \mathrm{~nm}$ diameter sample, $\sim 1.7$ times higher than that of $20 \mathrm{~nm}$ diameter sample, and $\sim 2$ times higher than that of the sample of streptavidin-conjugated Alexa Fluor $647(\sim 5 \mathrm{~nm}$ diameter). Unfortunately, the factor of 2 improvement in the modulation efficiency is limited compared to the factor of 200 increase in the size of fluorescent particles (from $5 \mathrm{~nm}$ to 1 micron). This result implies that the observed increases in modulation efficiencies from different research groups mentioned in the introduction section cannot be justified based solely on the size differences of the adopted fluorescent particles. The finding of the sizedependent modulation efficiency in this study implies that large fluorescent particles (microns) are easier to modulate compared with small fluorescent particles (nanometers). If one assumes that the major modulation mechanism is the modulation of fluorophore concentration as discussed in our previous studies $[10,13]$, the current results imply that for the same concentration of the fluorophore the larger microspheres can be modulated relatively more efficiently. Note that the upper limit of the size being effective in the modulation has not been specifically studied. However, the particle size is usually limited to be smaller than capillary diameter for biomedical imaging applications. Therefore, we limited our particle size to below one micron in this study.

The peak UMF signals from the three-sized fluorescent microsphere solutions (with the concentration of $1.0 \mathrm{mg} / \mathrm{mL}$ ) were measured as a function of the peak pressure applied at the transducer focus $\left(P_{\mathrm{fspp}}\right)$. The background electronic interference was measured at each $P_{\text {fspp }}$ value and subtracted from the corresponding UMF signal. Figure 6 shows a the linear relationships between the UMF and the $P_{\text {fspp }}$ for all the three samples. This result is consistent with our previous studies $[10,13]$ and indicates that the fluorescent particle size does not affect the linear relationship between the UMF strength and the ultrasound pressure [13].

\section{Summary}

In summary, modulation efficiencies from four differentsized fluorescent particles solutions (from $5 \mathrm{~nm}$ to $1 \mathrm{um}$ scale) were measured and compared. The modulation efficiency increases by approximately a factor of two when increasing the fluorescent particle size from 5 nanometers to 1 micron. This size-dependent modulation efficiency indicates that large fluorescent particles can be slightly more efficiently modulated. The mechanism may be that larger particles are ultrasonically oscillated more efficiently and larger particle can encapsulate more fluorescent molecules. However, the improvement is very limited and may not be considered as a strategy to significantly improve the modulation efficiency of UMF for biological imaging applications. This study also indicates that new modulation mechanisms should be explored to gain further improvements in modulation efficiency.

\section{Acknowledgments}

The authors acknowledge the funding support of National Institute of Health (7R15EB012312-02), CPRIT (RP120052) and seed grant support of Research Enhancement Program from University of Texas at Arlington

\section{References}

[1] W. Cai and X. Chen, "Multimodality molecular imaging of tumor angiogenesis," Journal of Nuclear Medicine, vol. 49, supplement 2, pp. 113S-128S, 2008.

[2] J. R. Lakowicz, Principles of Fluorescence Spectroscopy, Speinger, Berlin, Germany, 3rd edition, 2006.

[3] L. V. Wang, "Multiscale photoacoustic microscopy and computed tomography," Nature Photonics, vol. 3, no. 9, pp. 503$509,2009$.

[4] J. Culver, W. Akers, and S. Achilefu, "Multimodality molecular imaging with combined optical and SPECT/PET modalities," Journal of Nuclear Medicine, vol. 49, no. 2, pp. 169-172, 2008.

[5] B. Yuan, "Theoretical study of ultrasound-modulated fluorescence photon waves in turbid media," in Diffuse Optical Tomography and Fluorescence Diffuse Optical Tomography, PhD Dissertation, chapter 6, University of Connecticut, Connecticut, RI, USA, 2006.

[6] Y. Li, H. Zhang, C. Kim, K. H. Wagner, P. Hemmer, and L. V. Wang, "Pulsed ultrasound-modulated optical tomography using spectral-hole burning as a narrowband spectral filter," Applied Physics Letters, vol. 93, no. 1, Article ID 011111, 2008.

[7] T. W. Murray, L. Sui, G. Maguluri et al., "Detection of ultrasound-modulated photons in diffuse media using the photorefractive effect," Optics Letters, vol. 29, no. 21, pp. 25092511, 2004.

[8] G. Rousseau, A. Blouin, and J.-P. Monchalin, "Ultrasoundmodulated optical imaging using a powerful long pulse laser," Optics Express, vol. 16, no. 17, pp. 12577-12590, 2008.

[9] L. V. Wang, "Mechanisms of ultrasonic modulation of multiply scattered coherent light: an analytic model," Physical Review Letters, vol. 87, no. 4, Article ID 043903, 2001.

[10] B. Yuan, J. Gamelin, and Q. Zhu, "Mechanisms of the ultrasonic modulation of fluorescence in turbid media," Journal of Applied Physics, vol. 104, no. 10, Article ID 103102, 2008.

[11] M. Kobayashi, T. Mizumoto, Y. Shibuya, M. Enomoto, and M. Takeda, "Fluorescence tomography in turbid media based on acousto-optic modulation imaging," Applied Physics Letters, vol. 89, no. 18, Article ID 181102, 2006.

[12] B. Yuan, Y. Liu, P. M. Mehl, and J. Vignola, "Microbubbleenhanced ultrasound-modulated fluorescence in a turbid medium," Applied Physics Letters, vol. 95, no. 18, Article ID 181113, 2009.

[13] B. Yuan and Y. Liu, "Ultrasound-modulated fluorescence from rhodamine B aqueous solution," Journal of Biomedical Optics, vol. 15, no. 2, Article ID 021321, 2010.

[14] D. J. Hall, U. Sunar, and S. Farshchi-Heydari, "Quadrature detection of ultrasound-modulated photons with a gainmodulated, image-intensified, CCD camera," The Open Optics Journal, vol. 2, pp. 75-78, 2008.

[15] D. J. Hall, M. J. Hsu, S. Esener, and R. F. Mattrey, "Detection of ultrasound-modulated photons and enhancement with ultrasound microbubbles," Proceedings of SPIE, vol. 7190, article 71900L, 2009.

[16] J. H. Shin, Y. Jiang, B. Grabowski, J. Hurwitz, and Z. Kelman, "Substrate requirements for duplex DNA translocation by the 
eukaryal and archaeal minichromosome maintenance helicases," The Journal of Biological Chemistry, vol. 278, no. 49, pp. 49053-49062, 2003.

[17] H. Li, S. H. Park, J. H. Reif, T. H. LaBean, and H. Yan, "DNAtemplated self-assembly of protein and nanoparticle linear arrays," Journal of the American Chemical Society, vol. 126, no. 2, pp. 418-419, 2004. 

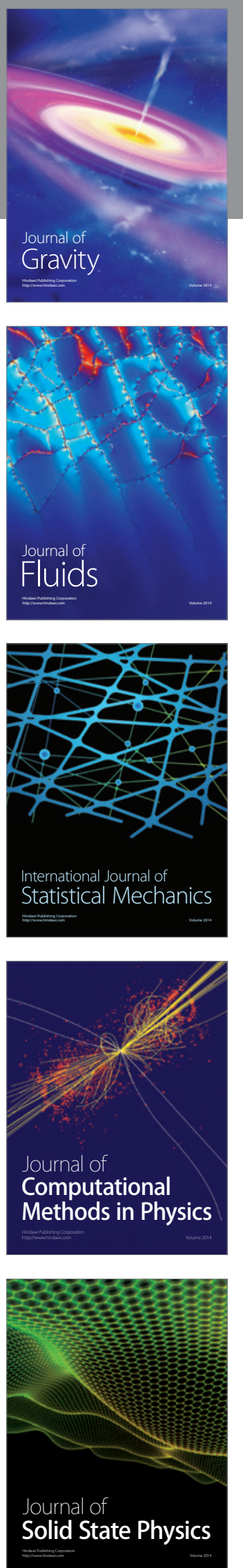

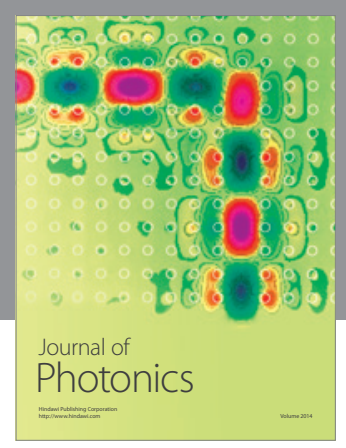

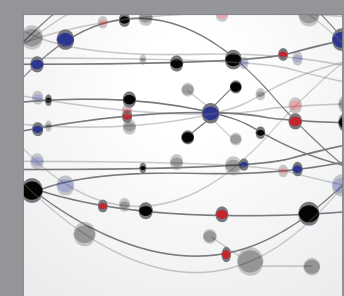

The Scientific World Journal
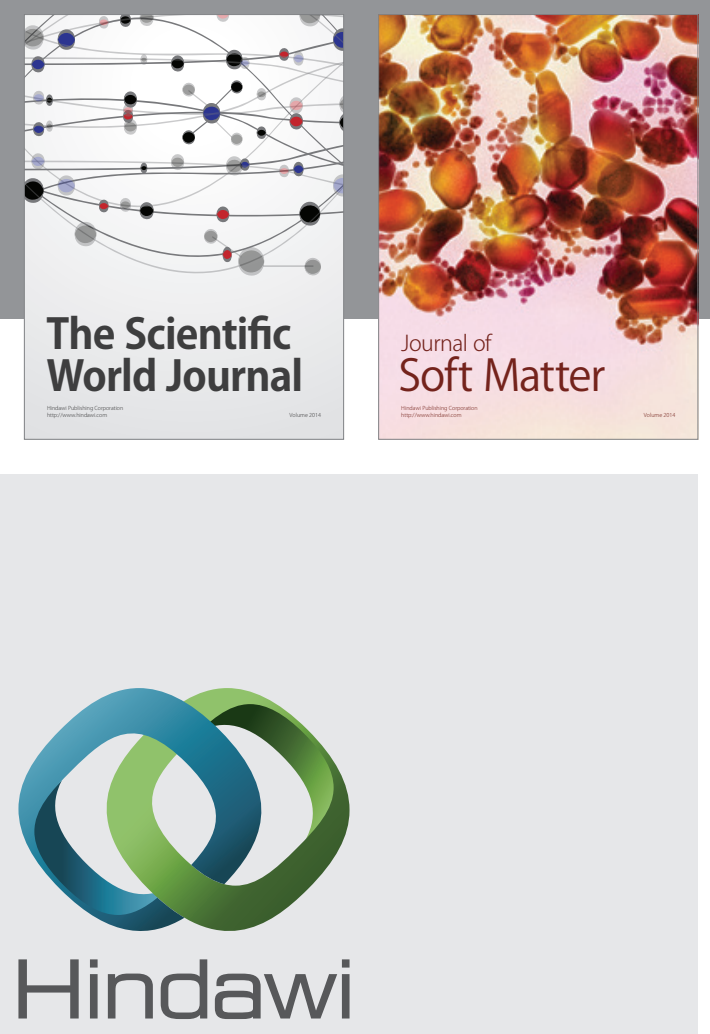

Submit your manuscripts at

http://www.hindawi.com
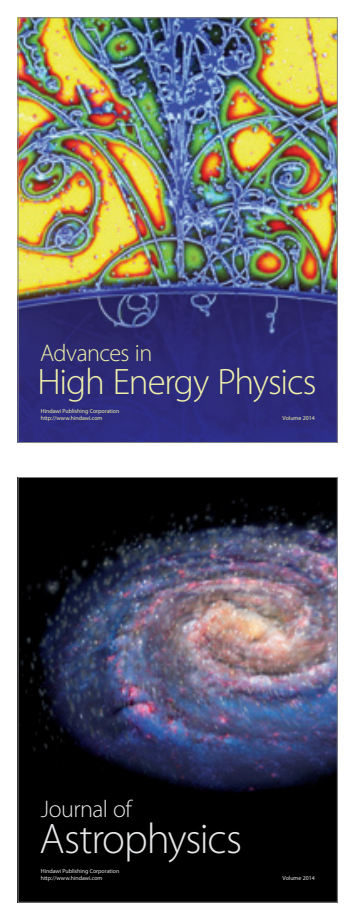
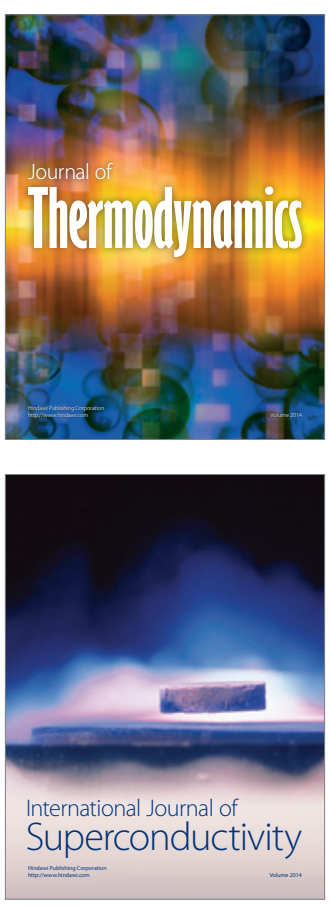
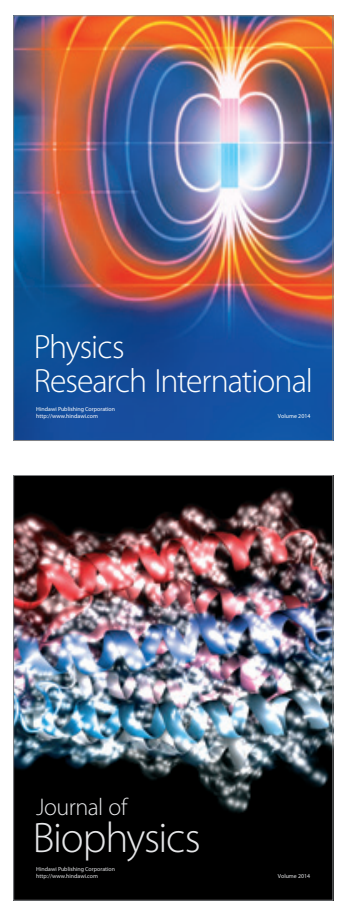
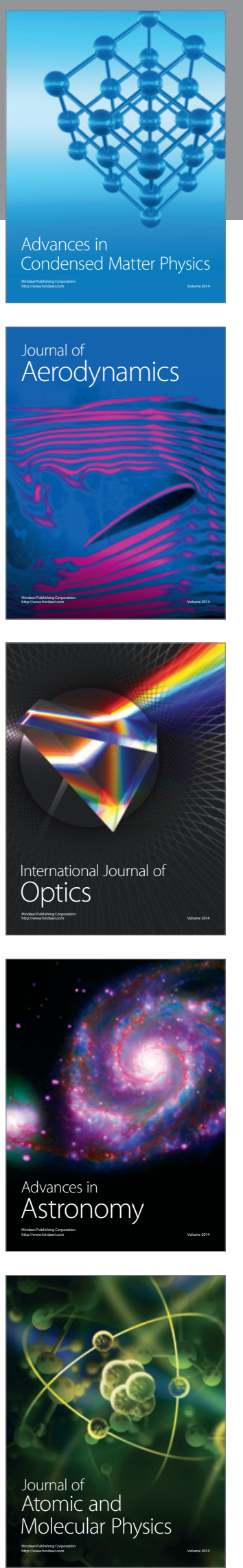